\title{
Mineração
}

\section{Impacto de diferentes métricas de risco na seleção de portfólios de projetos de produção de petróleo}

\author{
(The impact of different risk measures \\ in the portfolio selection of oil producing \\ projects)
}

\section{Rafael Costa Galeno}

Laboratório de Análises Geoeconômicas (LAGE). Faculdade de Engenharia Mecânica, Departamento de Engenharia de Petróleo, UNICAMP. E-mail:galeno@gmail.com

\section{Saul B. Suslick}

Instituto de Geociências e Centro de Estudos de Petróleo, UNICAMP. E-mail:suslick@ige.unicamp.br

\section{Márcio A. Sampaio Pinto}

Laboratório de Análises Geoeconômicas (LAGE). Faculdade de Engenharia Mecânica, Departamento de Engenharia de Petróleo, UNICAMP. E-mail:marcio@dep.fem.unicamp.br

\section{Gabriel Alves da Costa Lima \\ Instituto de Geociências e Centro de Estudos de Petróleo, UNICAMP. E-mail: gabriel@ige.unicamp.br}

\section{Resumo}

O processo de seleção de portfólios de projetos na exploração e produção de petróleo é uma tarefa bastante complexa, que passa por diferentes etapas até atingir o ponto final, que é a seleção do portfólio ótimo. A etapa de mensuração do risco do investimento abrange diferentes tipos de métricas com impactos diferenciados na escolha do portfólio. Esse trabalho apresenta a metodologia para a seleção de portfólios considerando o semidesvio-padrão como medida de risco. A metodologia é aplicada para um conjunto de seis projetos de produção de petróleo localizados na bacia de Campos, onde é feita a minimização do risco sujeito a um determinado retorno. Como ferramenta de otimização é utilizada a técnica de algoritmos genéticos. A seleção de portfólios foi feita pela maximização do equivalentecerto, para diferentes valores de coeficientes de aversão ao risco. Os resultados mostram que o semidesvio-padrão, apresenta maior sensibilidade na seleção de portfólios com projetos que apresentam grandes magnitudes de risco e retorno.

Palavras-chave: Semidesvio-padrão, teoria do portfólio, teoria da preferência, petróleo.

\section{Abstract}

The portfolio selection process for projects involving the exploration and production of oil is a complex task, passing through different steps until and optimum solution is achieved, revealing the best portfolio. This paper presents a methodology for portfolio selection considering semi-standard deviation as a measure of risk. The methodology is applied to a set of six oil production projects located in the Campos Basin, where the risk minimization is subject to a certain return. Genetic algorithms are used as the optimization tool.. The portfolio selection was performed by maximization of the certainty equivalent, for different values of risk aversion coefficients. The results show that the semi-standard deviation presents better sensitivity in portfolio selection with projects that have a large magnitude of risk and return.

Keywords: Semi-standard deviation, portfolio theory, preference theory, petroleum. 


\section{Introdução}

A solução do problema da estimativa de risco das oportunidades de investimento é uma das principais contribuições para que uma empresa se torne competitiva - assumir riscos desnecessários implica elevar a possibilidade de perdas financeiras, enquanto que evitar riscos de forma indiscriminada poderá acarretar a rejeição de oportunidades de investimentos.

No segmento de exploração e produção (E\&P) de petróleo, o problema de identificação das variáveis mais críticas, tais como a modelagem da incerteza e a quantificação do risco, constitui importante fator de analistas e tomadores de decisão nesse segmento.

Em muitos casos, o risco ganha mais importância em função das características financeiras, econômicas e operacionais. No caso de E\&P de petróleo, algumas características que se destacam são:

- Os projetos de E\&P requisitam intensos volumes de capital.

- Os projetos possuem longos períodos de maturação, que podem chegar a mais de 10 anos (Siddiqui, 2007; Petrobras, 2008).

- A volatilidade dos preços, os custos crescentes, as demandas atuais e futuras dos produtos derivados de petróleo apresentam incertezas e riscos nas suas diferentes etapas, com impacto direto nos estudos de viabilidade econômica dos projetos e na construção de suas carteiras (Suslick \& Schiozer, 2004).

Um problema importante consiste em escolher a medida de risco da taxa de retorno dos projetos. Classicamente, métricas como o desvio-padrão ou a variância vêm sendo utilizadas, mas essas métricas apresentam limitações no sentido de que elas quantificam apenas a dispersão em torno da média e, assim, penalizam, igualmente, tanto os retornos positivos, quanto os negativos. Ocorre que, na indústria de petróleo, em virtude dos altos investimentos, uma escolha de medida de risco inadequada pode levar à realização de escolhas incompatíveis com o perfil da empresa em relação às suas decisões sob risco.

A partir da escolha da medida de risco de cada um dos projetos, a próxima fase consiste em realizar uma análise envolvendo os diversos projetos que formam o portfólio de ativos de produção da empresa. Dessa forma, como mostram Costa Lima e Suslick (2005), na tomada de decisão, na indústria de petróleo, os profissionais devem utilizar uma combinação de ferramentas e metodologias, que podem incluir técnicas de otimização, teoria moderna do portfólio, análise de risco, teoria da preferência, entre outras.

No caso da teoria do portfólio de Markowitz (1952), o seu produto final é a fronteira eficiente, ferramenta que é muito útil para que o tomador de decisão possa visualizar, simultaneamente, tanto a capacidade de geração de retorno, como a exposição ao risco. Classicamente, na construção da fronteira eficiente, utiliza-se o desvio-padrão como a métrica de risco. No entanto, se utilizarmos outros indicadores, para quantificar o risco, a fronteira eficiente será diferente. $\mathrm{O}$ objetivo desse artigo é estudar o comportamento da fronteira eficiente utilizando como métrica de risco o semidesviopadrão.

Esse artigo está organizado em seis seções. Na primeira, são discutidas a análise e a seleção dos portfólios. Na segunda, são abordadas as métricas de risco e de retorno dos projetos. Na terceira, são discutidas as métricas de retorno e risco de portfólios de projetos. Na quarta, será dada uma visão geral sobre a escolha do portfólio ótimo. Na quinta, é apresentado um estudo de caso utilizando a metodologia abordada e, na última seção, realiza-se uma discussão dos resultados obtidos, seguidos das conclusões.

\section{Indicadores para quantificar o retorno e o risco de projetos}

Quando pensamos em indicadores para retorno e risco de projetos, inicial- mente devemos considerar que a variável retorno (VPL, TIR, etc.) é aleatória e, por isso, os seus valores reais não podem ser conhecidos com certeza, mas apenas estimados usando modelos probabilísticos.

O retorno de um projeto (digamos, o seu VPL) é dado por um indicador econômico, que depende de diversas variáveis, tais como preço, demanda, custo, tributos, custo de capital, entre outras. Portanto, para estimar o VPL, devemos realizar projeções de todas essas variáveis incertas ao longo de um horizonte de tempo, que pode alcançar, não raramente, cerca de 20 anos. Tais projeções são realizadas, geralmente, usando-se modelos de regressão e/ou séries temporais, juntamente com simulação de Monte Carlo. Por meio de simulação de Monte Carlo, podemos gerar milhares de trajetórias distintas para preços, custos, taxas de juros, etc. Uma vez que todas as trajetórias são equiprováveis, podemos utilizar a média aritmética por meio de:

$E\left[V P L_{p r o}\right]=\frac{\sum_{i=1}^{N} V P L_{i}}{N}$,

onde $V P L_{i}$ se refere ao valor gerado pela i-ésima simulação e $N$ é o número total de simulações.

Depois de estimar a média aritmética, podemos estimar o desvio-padrão da distribuição de probabilidade do VPL de cada projeto por meio de:

$\sigma_{\text {pro }}=\sqrt{\frac{\sum_{i=1}^{N}\left(V P L_{i}-E\left[V P L_{p r o}\right]\right)^{2}}{N}}$

sendo que o desvio-padrão é a medida mais usada para a quantificação de risco (Walls, 2004; Campbell, Lo \& MacKinlay, 1997), tanto para análise em mercado financeiro, como na engenharia econômica.

A Equação (2) considera que são igualmente indesejáveis, tanto os valores abaixo, como acima da média e isso pode não ser coerente com os objetivos dos tomadores de decisão, pois, geralmente, a preocupação é com as perdas, 
que se tornam mais graves no caso de distribuições assimétricas. Alternativamente, podemos usar um indicador que considere somente a dispersão de valores no lado esquerdo da distribuição, ou seja, o semidesvio-padrão, dado por:

$\sigma_{\text {pro }}=\sqrt{\frac{\sum_{i=1}^{n}\left(\min \left[0, x_{i}-b\right]\right)^{2}}{n}}$

onde $\sigma_{p r o}$ é o semidesvio-padrão do projeto, $x_{i}$ são os valores do VPL, gerados pela simulação de Monte Carlo, e $b$ é o ponto crítico, abaixo do qual o investidor se preocupa, e $n$ é o número de simulações.

$\mathrm{O}$ valor de $b$ representa o mínimo retorno aceitável, ou seja, o ponto no qual a dispersão da distribuição à esquerda é medida. Para ilustrar, nas Figuras 1(a) e 1(b), encontram-se ilustrações do desvio-padrão e do semidesvio-padrão.

O conceito de semidesvio-padrão não é novo e suas aplicações, na área de finanças, surgiram com Markowitz (1959) que, em seu livro clássico, observa que a escolha entre as duas medidas depende da conveniência, familiaridade, diferenças entre os portfólios produzidos por diferentes métricas, entre outras características pertinentes.
Uma característica importante a ser ressaltada é que o valor numérico do desvio-padrão é, no mínimo, igual ao do semidesvio-padrão. A implicação imediata é que não podemos fazer comparação entre o desvio-padrão e o semidesvio-padrão, muito embora os dois possuam unidades iguais.

\section{Indicadores para quantificar o retorno e o risco de portfólios de projetos}

No caso em que a análise se referir a um portfólio de projetos, o retorno do portfólio será igual a:

$V P L_{p}=\sum_{i=1}^{n} V P L_{\text {pro }_{i}} x_{i}$

onde $V P L_{p}$ é o retorno do portfólio, $V P L_{p r o i}$ é o valor da média aritmética do VPL do projeto e $x_{i}$ é o nível de participação no projeto i. Veja que o VPL de um portfólio de projetos é uma grandeza aditiva.

Uma vez que o VPL dos projetos é uma variável aleatória, podemos estimar o seu desvio-padrão por meio de:

$V P L_{p}=\sqrt{\sum_{i=1}^{n} x_{i}^{2} \sigma_{i}^{2}+\sum_{i=1}^{n} \sum_{\substack{j=1 \\ j \neq i}}^{n} x_{i} x_{j} \sigma_{\text {pro }_{i}} \sigma_{\text {pro }} \rho_{i, j}}$

onde $\sigma_{p}$ é o risco do portfólio, $\rho_{i, j}$ é a correlação entre os projetos i e j, $\sigma_{p r o i}$ é o desviopadrão do projeto i e j.

O uso da Equação (5) nem sempre é fácil, porque depende do coeficiente de correlação entre os valores dos VPLs de cada par de projetos. Mas não podemos conhecer a correlação futura dos projetos a não ser por meio de simulação, uma vez que não há séries históricas de VPLs de projetos, como ocorre no caso de ativos financeiros.

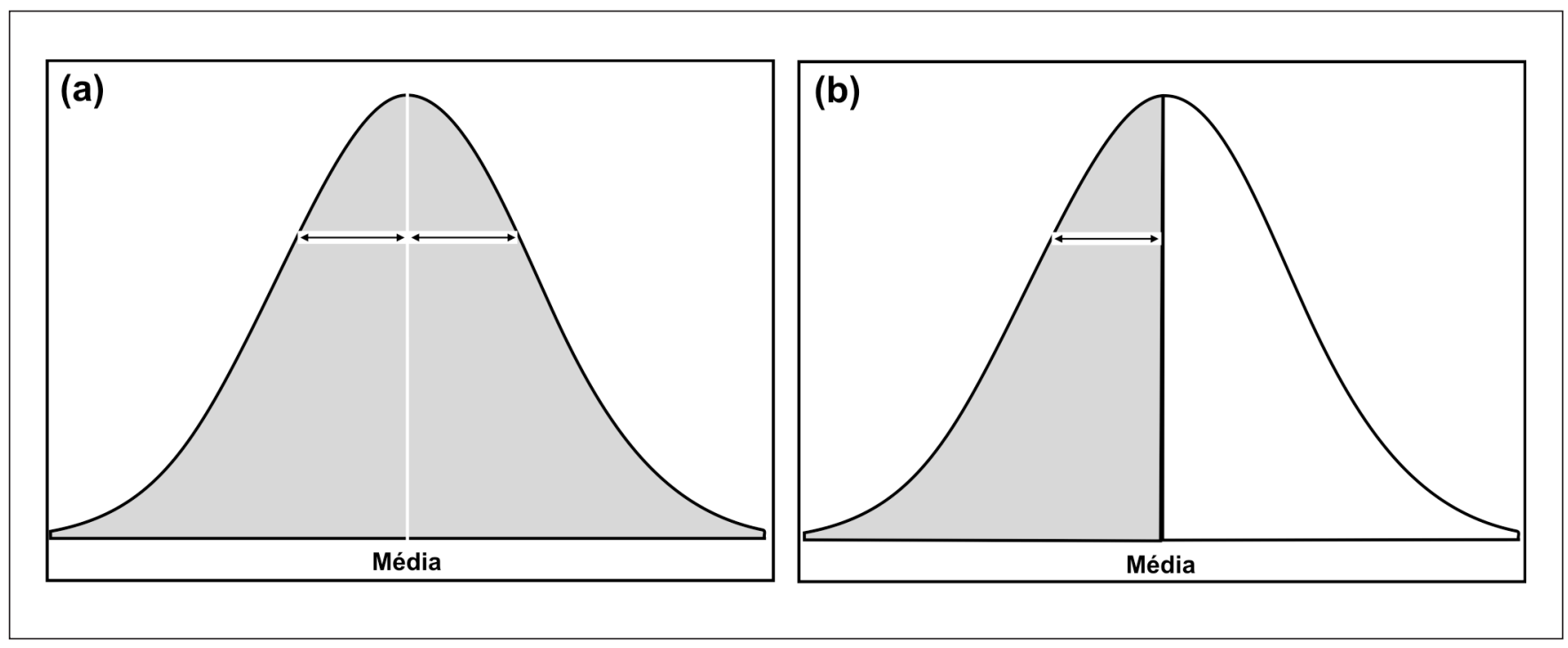

Figura 1 - Ilustração gráfica do ( a) desvio-padrão e do (b) semidesvio-padrão. 
Nas Equações (4) e (5), a flexibilidade do gerente consiste em escolher o valor de $x_{i}$, que se refere ao nível de participação em cada um dos projetos. A partir de diferentes valores de $x_{i}$ (alocação dos recursos), pode-se obter o principal produto do modelo de Markowitz, que é o que se chama de fronteira eficiente, ou seja, a curva geométrica composta por um número infinito de portfólios eficientes (Costa Lima, Schiozer \& Suslick 2006).

Para ilustrar o conceito de fronteira eficiente, consideremos um portfólio composto por dois projetos $\mathrm{A}$ e $\mathrm{B}$, que se encontram na Tabela 1.

Na Tabela 1, o risco é definido como o desvio-padrão dos VPLs dos projetos A e B. Uma escolha baseada somente no retorno indicaria o projeto $\mathrm{B}$, mas isso implica aceitar um risco superior ao do projeto A.

A partir dos projetos A e B, podemos formar infinitos portfólios compostos por participações nesses ativos. No entanto, nesse artigo, foi considerado que os níveis de participação da empresa, em cada projeto, possuem um incremento igual a $5 \%$. O resultado final pode ser visualizado na forma de um gráfico, que se chama fronteira eficiente e se encontra na Figura 2.

$\mathrm{Na}$ Figura 2, encontram-se somente os portfólios da fronteira eficiente, muito embora existam muitos outros. Um portfólio é dito eficiente, se não existir nenhum outro portfólio que tenha maior retorno, para o mesmo risco ou menor, e se não existir nenhum outro portfólio que tenha menor risco, para o mesmo retorno ou maior.

A construção da fronteira eficiente representa um progresso importante no processo de tomada de decisões, mas o decisor deve escolher qual é o melhor portfólio entre aqueles que se encontram na fronteira eficiente. A solução desse problema nem sempre é simples, pois depende de uma série de considerações, tais como estratégia da empresa, nível de diversificação dos acionistas, hori-

\begin{tabular}{c|c|c}
\hline Tabela 1 - Valores de risco e retorno dos projetos A e B. \\
\hline & Projeto A & Projeto B \\
\hline Retorno (Milhões US\$) & 94,18 & 415,12 \\
\hline Risco (Milhões US\$) & 175,25 & 424,05 \\
\hline
\end{tabular}

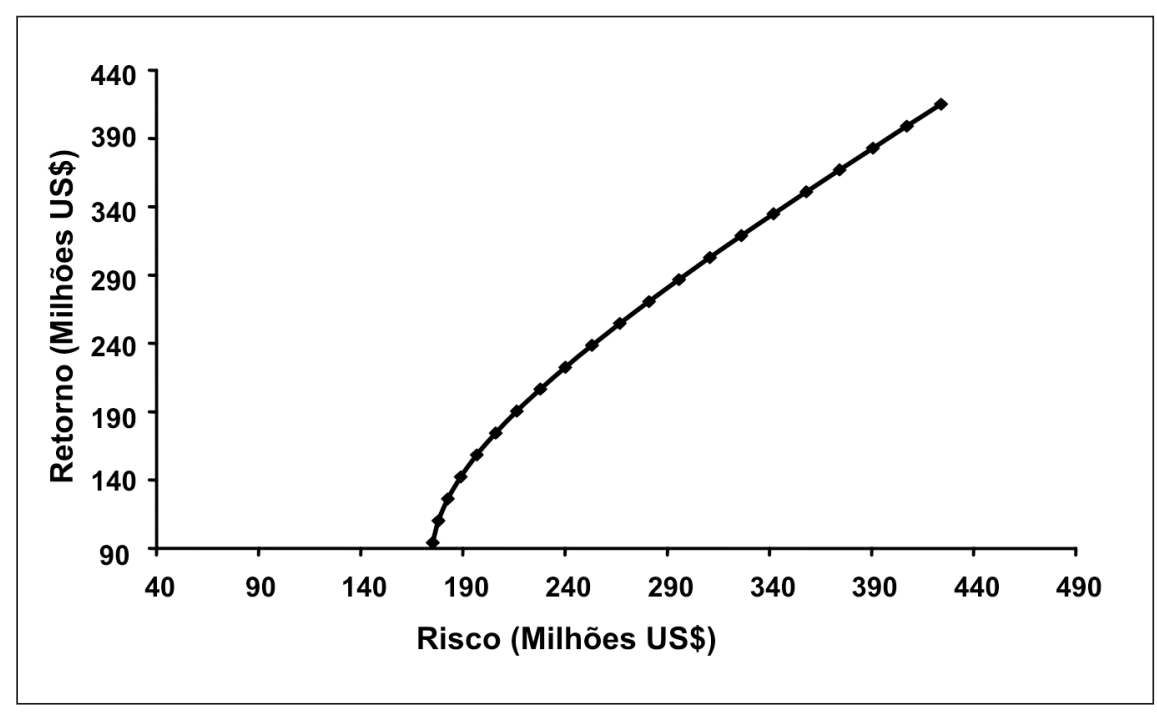

Figura 2 - Fronteira eficiente de dois projetos.

zonte das expectativas das empresas, entre outros determinantes. Na próxima seção, vamos considerar o problema da escolha do portfólio ótimo.

\section{A escolha do portfólio ótimo a partir da fronteira eficiente}

A escolha do portfólio ótimo, sob a ótica de um investidor diversificado, é imediata: escolher o portfólio com maior retorno sem levar em consideração o seu risco. Mas, por outro lado, no caso de um investidor não-diversificado, a escolha é diferente, pois ele tem que assumir frações de riscos, que poderiam ser reduzidas simplesmente por meio da diversificação.

A teoria da utilidade, desenvolvida por Von Neumann e Morgenstern (1953), pode ser usada para orientar a escolha sob incerteza, tal como ocorre na seleção do portfólio ótimo, a partir da fronteira eficiente. A função utilidade exponencial é dada por:
$U(x)=a-b e^{-\frac{x}{R}}$

onde $a$ e $b$ são constantes, $R$ deve ser entendido como um valor de tolerância ao risco e $x$ é o valor monetário, sendo que $x$ e $R$ devem ter as mesmas unidades. O uso da função exponencial na tomada de decisões na indústria de petróleo aparece em Cozzolino (1980), Walls (1995) e Nepomuceno e Suslick (2000).

Uma simplificação, que aparece freqüentemente, consiste em fazer $a=0$ e $b=1$, na equação (6), como fizeram Nepomuceno e Suslick (2000). O uso dessa equação, para realizar previsões, depende da estimativa dos seus parâmetros, sobretudo $R$. Em um trabalho empírico, realizado para a exploração de petróleo, Walls e Dyer (1996) relacionaram o valor de $R$ ao montante do orçamento da empresa e concluíram que $R$ fica em torno de $25 \%$ do orçamento anual da exploração.

Como mostra Costa Lima, Schiozer e Pereira (2006), o valor da função utilidade não possui significado prático, 
Rafael C. Galeno et al.

quando analisado isoladamente, pois é apenas um número que reflete as preferências de um indivíduo por um determinado investimento. Por isso, devemos usar uma grandeza que possui a mesma unidade que o VPL, tal como o chamado equivalente-certo (EC). O equivalentecerto é definido por meio de:

$$
E[U(E C)]=E\left[\sum_{i=1}^{n} p_{i} \cdot U\left(x_{i}\right)\right]
$$

A interpretação da Equação (7) é importante. $\mathrm{O}$ valor esperado da utilidade do equivalente-certo é igual ao valor esperado das utilidades de todos os resultados possíveis, ou seja, o equivalente-certo é uma quantidade cujo valor numérico é igual ao valor esperado da soma das utilidades de todos os eventos. Pode-se afirmar que o equivalentecerto é um valor sobre o qual inexiste risco, mas com valor de utilidade igual ao valor de utilidade gerado por $N$ eventos incertos.

No caso do VPL de um projeto que é uma variável aleatória, o seu valor verdadeiro somente será conhecido ao final do projeto. Mas, na prática, é possível estimar qual seria a distribuição de probabilidade do VPL, ao discretizá-la e, a partir daí, estimar qual seria o valor de utilidade do equivalente-certo:

$$
U(E C)=\sum_{i=1}^{n} p_{i} \cdot E\left[U\left(V P L_{i}\right)\right]
$$

Uma questão importante é que a aversão ao risco (ou tolerância ao risco) depende, principalmente, da relação entre o montante exposto ao risco e magnitude de riqueza do tomador de decisão. Por isso, podemos pensar na utilidade não exatamente do $E C$, mas, sim, de um indicador como o $E C / R$, onde $R$ é novamente a tolerância ao risco do tomador de decisão. Assim, a Equação (8) se transforma em:

$$
-e^{-\frac{E C}{R}}=\sum_{i=1}^{n}\left[p_{i} \cdot\left(-e^{-\frac{V P L_{i}}{R}}\right)\right]
$$

A partir da Equação (9), depois de alguma manipulação algébrica, pode-se chegar à seguinte expressão:

$$
E C=-R \cdot \ln \left[\sum_{i=1}^{n}\left(p_{i} \cdot e^{-\frac{V P L_{i}}{R}}\right)\right]
$$

A Equação (10) pode ser utilizada para comparar alternativas de investimento que possuam diferentes níveis de retorno e risco. É importante observar que essa equação possui aplicabilidade geral para qualquer função de densidade de probabilidade da variável aleatória do VPL. No entanto, se a distribuição do VPL for Gaussiana e a função de utilidade for do tipo exponencial ( $\operatorname{com} a=0 \mathrm{e}$ $b=1$ ), Galeno (2008) mostra que o equivalente-certo pode ser calculado por:

$$
E C=\mu_{V P L}-\frac{\sigma_{V P L}^{2}}{2 R}
$$

onde $\mu_{V P L}$ e $\sigma_{V P L}$ são a média e o desviopadrão da distribuição normal usada na modelagem do VPL, respectivamente. Veja que o equivalente-certo é uma grandeza monetária e, assim, possui a mesma natureza do VPL.

A Equação (11) pode ser usada para estimar o valor do equivalente-certo, tanto de projetos que sejam analisados individualmente, como de portfólios de projetos. Ela é derivada a partir dos princípios da teoria da escolha sob incerteza (também conhecida com teoria da preferência) e aparece, na literatura de análise de decisão sob incerteza, em diversos trabalhos como os de Holloway (1979), Cozzolino (1980), Luenberger (1998), Nepomuceno \& Suslick (2000) e Costa Lima, Schiozer e Suslick (2006). A escolha entre dois projetos deve ser pelo projeto de maior equivalente-certo.

Alternativamente, pode-se usar o semidesvio-padrão como medida de risco dos projetos e estimar o valor do equivalente-certo, como fizeram Campbell, Campbell e Campbell (2001) e Kritzman (2003). Esses trabalhos mostram que no caso da função de utilidade exponencial, o equivalente-certo pode ser estimado por meio de:
$E C=\mu_{V P L}-r_{T} \sigma_{V P L}^{2}-r_{S V} \sigma_{S V}^{2}$

onde $\mu_{V P L}$ é o retorno do portfólio, $\sigma_{V P L}$ é o desvio-padrão do portfólio, $\sigma_{S V}$ é o semidesvio-padrão do portfólio, $r_{T}$ é o coeficiente de aversão ao risco do desvio-padrão e $r_{S V}$ é o coeficiente de aversão ao risco do semidesvio-padrão. Os modelos de estimativa do equivalente certo que aparecem nas Equações (11) e (12) serão usados para escolher o melhor portfólio de projetos de produção de petróleo.

\section{Seleção do portfólio ótimo usando a teoria do equivalente certo}

Consideremos o caso de uma empresa fictícia denominada Beta Petróleo $\mathrm{S} / \mathrm{A}$, uma multinacional que atua na área de produção de petróleo e que possui diversas oportunidades de investimentos com as seguintes características:

- Seis projetos hipotéticos de produção marítima de petróleo localizados na bacia de Campos.

- Os volumes de hidrocarbonetos foram determinados pela interpretação de dados sísmicos e confirmados por poços pioneiros de avaliação e testes de formação.

- A vida operacional varia entre 11 a 22 anos, o que é padrão para a indústria de E\&P de petróleo atuando nessa bacia marítima.

Inicialmente, foram realizadas análises econômicas nesse conjunto de projetos de modo a estimar o VPL e o risco, usando, tanto o desvio-padrão, como o semidesvio-padrão. Os resultados que se encontram na Tabela 2 foram obtidos usando-se a ferramenta de simulação de Monte Carlo.

Como pode ver visto na Tabela 2, os projetos da empresa possuem características bastante distintas em termos de VPL, risco (tanto em termos de desvio-padrão, como de semidesvio-padrão) e investimento. 
Para a escolha do portfólio, uma consideração importante é que a empresa não deseja alocar mais que $70 \%$ do seu orçamento (US\$ 1,12 bilhão) no desenvolvimento dos novos projetos. Há outras variáveis que devem ser consideradas, tais como restrições de fluxo de caixa, de produção de óleo e de água, entre outras restrições. Entretanto, com a intenção de simplificar o modelo, será considerada, apenas, a restrição de investimento:

$$
\sum_{i=1}^{n} x_{i} \cdot I_{i}(t) \leq I_{i}^{*}(t)
$$

onde $x_{i}$ é o nível de participação no projeto $\mathrm{i}, I_{i}(t)$ é o investimento em função do tempo de cada projeto e $I_{i}^{*}(t)$ é a restrição de investimento em função do tempo da empresa.

Uma variável importante do modelo de otimização é estimada por meio da correlação entre os VPLs de cada par de projetos de produção de petróleo, mas a estimativa dessas correlações está fora do escopo desse trabalho. Por isso, vamos assumir que a correlação entre os projetos seja igual a 0,70 , ou seja, há uma chance de $70 \%$ de que cada par de projetos tenha VPLs cujos valores se movimentam na mesma direção ao longo do tempo.

Nesse modelo de seleção de portfólios, será considerado que a prin- cipal flexibilidade do tomador de decisão consiste na escolha do nível de participação em cada um dos projetos que compõe o portfólio da empresa e que este se encontra entre $0 \%$ a $100 \%$. Na Tabela 3 , encontram-se os resultados dos valores ótimos dos níveis de participação que foram obtidos após a realização da otimização utilizando-se algoritmos genéticos.

A Tabela 3 apresenta 19 portfólios gerados e cada um deles possui diferentes composições, em termos de níveis de participação de cada um dos projetos, bem como em termos de VPLs e risco (desvio-padrão ou de semidesvio-padrão). De maneira geral, as tendências mostram que na medida em que se aumenta a construção de um portfólio com maior VPL, o risco tende a aumentar. Portanto, a escolha do melhor portfólio não é simples, uma vez que, se o tomador de decisão buscar aumentar muito o retorno implicará elevar, excessivamente, o risco. Logo a escolha deve considerar variáveis como: nível de risco do portfólio, tolerância ao risco da empresa e nível de aversão ao risco do tomador de decisão. Para isto, podem ser utilizadas as Equações (11) e (12) para a estimativa do equivalente certo.

A estimativa da tolerância ao risco não é simples, uma vez que depende de características de cada tomador de decisão, bem como do tamanho da empresa. Um trabalho empírico de Walls (1995) estimou que o coeficiente de aversão ao risco das empresas de petróleo se situava, naquela data, em torno de $25 \%$ do orçamento para exploração de petróleo.

No entanto, a fase de produção ainda carece de uma pesquisa empírica para encontrar a tolerância ao risco dos tomadores de decisão, pois os montantes financeiros sujeitos à incerteza apresentam magnitudes distintas. Em virtude disso, nesse trabalho, será considerado que a tolerância ao risco se encontra em torno de $25 \%$ do orçamento das empresas para investimento em novos projetos. Os resultados se encontram na Tabela 4 .

$\mathrm{Na}$ Tabela 4, encontram-se 19 portfólios, sendo que, para cada um deles, foram estimados o VPL, o risco medido pelo desvio-padrão do VPL e pelo semidesvio-padrão do VPL.

Uma vez que as informações disponíveis referentes à tolerância ao risco são escassas, na fase de produção, vamos realizar uma análise de sensibilidade do EC, em relação a esses dois indicadores. No caso da estimativa do EC, usando-se o desvio-padrão como medida de risco, foram considerados dois valores para a tolerância ao risco: US\$ 300 milhões e US\$ 350 milhões. Já, para o caso de estimativa da função de utilidade, usando-se o semidesvio-padrão, devemos considerar dois parâmetros: a tolerância ao risco, considerando-se o desvio-padrão como medida de risco

Tabela 2 - Resultado do VPL e risco das oportunidades de investimento nos diferentes portfólios de projetos da Beta Petróleo S/A.

\begin{tabular}{c|c|c|c|c}
\hline \multirow{2}{*}{ Projetos } & VPL & \multicolumn{2}{c|}{ Risco (Milhões US\$) } & Investimento \\
\cline { 2 - 5 } & (Milhões US\$) & Desvio-padrão & Semidesvio-padrão & (Milhões US\$) \\
\hline Projeto 01 & 335,35 & 366,10 & 257,84 & 400 \\
\hline Projeto 02 & 356,25 & 260,73 & 184,31 & 150 \\
\hline Projeto 03 & 93,40 & 178,26 & 125,95 & 100 \\
\hline Projeto 04 & 364,92 & 313,82 & 221,50 & 350 \\
\hline Projeto 05 & 463,48 & 361,61 & 255,05 & 450 \\
\hline Projeto 06 & 181,33 & 212,04 & 149,95 & 150 \\
\hline
\end{tabular}


Rafael C. Galeno et al.

Tabela 3 - Resultados dos níveis de participação obtidos para os diferentes projetos.

\begin{tabular}{|c|c|c|c|c|c|c|c|c|c|}
\hline \multirow{2}{*}{ Portfólio } & \multirow{2}{*}{$\begin{array}{c}\text { Retorno } \\
\text { (Milhões } \\
\text { US\$) }\end{array}$} & \multirow{2}{*}{$\begin{array}{c}\text { Risco } \\
\text { (Desvio-padrão) } \\
\text { (Milhões US\$) }\end{array}$} & \multirow{2}{*}{$\begin{array}{c}\text { Risco (Semi- } \\
\text { desvio-padrão) } \\
\text { (Milhões US\$) }\end{array}$} & \multicolumn{6}{|c|}{ Nível de participação } \\
\hline & & & & Proj 1 & Proj 2 & Proj 3 & Proj 4 & Proj 5 & Proj 6 \\
\hline A1 & 100 & 69,23 & 48,89 & $0 \%$ & $16 \%$ & $0 \%$ & $3 \%$ & $8 \%$ & $0 \%$ \\
\hline A2 & 150 & 103,84 & 73,33 & $0 \%$ & $23 \%$ & $0 \%$ & $4 \%$ & $11 \%$ & $0 \%$ \\
\hline A3 & 200 & 138,45 & 97,78 & $0 \%$ & $31 \%$ & $0 \%$ & $5 \%$ & $15 \%$ & $0 \%$ \\
\hline A4 & 250 & 173,06 & 122,22 & $0 \%$ & $39 \%$ & $0 \%$ & $7 \%$ & $19 \%$ & $0 \%$ \\
\hline A5 & 300 & 207,68 & 146,67 & $0 \%$ & $47 \%$ & $0 \%$ & $8 \%$ & $23 \%$ & $0 \%$ \\
\hline A6 & 350 & 242,29 & 171,11 & $0 \%$ & $54 \%$ & $0 \%$ & $9 \%$ & $27 \%$ & $0 \%$ \\
\hline A7 & 400 & 276,90 & 195,56 & $0 \%$ & $62 \%$ & $0 \%$ & $11 \%$ & $30 \%$ & $0 \%$ \\
\hline A8 & 450 & 311,51 & 220,00 & $0 \%$ & $70 \%$ & $0 \%$ & $12 \%$ & $34 \%$ & $0 \%$ \\
\hline A9 & 500 & 346,13 & 244,45 & $0 \%$ & $78 \%$ & $0 \%$ & $13 \%$ & $38 \%$ & $0 \%$ \\
\hline A10 & 550 & 380,74 & 268,89 & $0 \%$ & $85 \%$ & $0 \%$ & $14 \%$ & $42 \%$ & $0 \%$ \\
\hline A11 & 600 & 415,35 & 293,34 & $0 \%$ & $93 \%$ & $0 \%$ & $16 \%$ & $46 \%$ & $0 \%$ \\
\hline A12 & 650 & 449,97 & 317,78 & $0 \%$ & $100 \%$ & $0 \%$ & $17 \%$ & $50 \%$ & $0 \%$ \\
\hline A13 & 700 & 484,88 & 342,41 & $0 \%$ & $100 \%$ & $0 \%$ & $22 \%$ & $57 \%$ & $0 \%$ \\
\hline A14 & 750 & 520,14 & 367,28 & $0 \%$ & $100 \%$ & $0 \%$ & $27 \%$ & $64 \%$ & $0 \%$ \\
\hline A15 & 800 & 555,69 & 392,35 & $0 \%$ & $100 \%$ & $0 \%$ & $31 \%$ & $71 \%$ & $0 \%$ \\
\hline A16 & 850 & 591,47 & 417,60 & $0 \%$ & $100 \%$ & $0 \%$ & $36 \%$ & $78 \%$ & $0 \%$ \\
\hline A17 & 900 & 627,45 & 442,98 & $0 \%$ & $100 \%$ & $0 \%$ & $41 \%$ & $85 \%$ & $0 \%$ \\
\hline A18 & 950 & 663,60 & 468,48 & $0 \%$ & $100 \%$ & $0 \%$ & $45 \%$ & $92 \%$ & $0 \%$ \\
\hline A19 & 1000 & 699,88 & 494,07 & $0 \%$ & $100 \%$ & $0 \%$ & $50 \%$ & $100 \%$ & $0 \%$ \\
\hline
\end{tabular}

(US\$ 750 e US\$ 900 milhões) e a tolerância ao risco considerando-se o semidesvio-padrão (US\$ 500 e US\$ 600 milhões). Os resultados encontram-se na Figura 3.

No caso da escolha recair no desvio-padrão como medida de risco, a preferência depende da tolerância ao risco do tomador de decisões. Se a tolerância ao risco for de US\$ 300 milhões, a escolha deve ser o portfólio A12, enquanto que, se a tolerância ao risco for de US\$ 350 milhões, a escolha deve ser pelo portfólio A13. Isto mostra que, ao se aumentar o valor da tolerância ao risco, o valor do equivalente-certo, sistematicamente, tende a aumentar também em todos os casos. Um comportamento semelhante se verifica no caso em que a estimativa do equivalente-certo é obtida por meio do semidesvio-padrão. No entanto, observa-se que não se pode comparar os valores de dois equivalentes-certo obtidos a partir do desvio-padrão e do semidesvio-padrão.

Os resultados obtidos para a seleção de portfólios a partir da aplicação da teoria do equivalente-certo são coerentes com a hipótese de aversão ao risco.
No caso de portfólios com baixo VPL e baixos riscos, o valor do equivalentecerto é baixo, em virtude da tolerância ao risco do tomador de decisão, o qual busca obter maior retorno. Já, no caso de portfólios com altos retornos, os altos riscos fazem com que o equivalente-certo seja baixo, em virtude do valor finito da tolerância ao risco do tomador de decisões. Dessa forma, haverá uma combinação de retorno e risco, que maximiza o equivalente-certo e será exatamente essa combinação que se busca na seleção do melhor portfólio. 
Impacto de diferentes métricas de risco na seleção de portfólios de projetos de produção de petróleo

\section{Conclusões}

Nesse artigo, foi discutido o problema da seleção do melhor portfólio entre aqueles que constituem a fronteira eficiente. No caso em que o tomador de decisão seja diversificado, a escolha é simples: selecionar o portfólio com maior retorno, independentemente do nível de risco. No entanto, no caso em que o tomador de decisão não seja diversificado (caso mais comum quando esse tomador de decisão se refere a uma empresa e que nem sempre ocorre diversificação, como é o caso de uma empresa integrada de petróleo), a seleção do melhor portfólio pode ser feita através da maximização do equivalente-certo.

A escolha da medida de risco (desvio-padrão ou semidesvio-padrão) possui forte impacto sobre o processo de escolha do portfólio ótimo. No caso da utilização do desvio-padrão, a escolha seria pelo portfólio A12 ou A13, e isto vai depender do nível de tolerância ao risco. No caso do emprego do semidesvio-padrão, a escolha seria entre os portfólios A8 e A10, dependendo, novamente, dos níveis de tolerância ao risco associados ao desvio e ao semidesviopadrão. Esse modelo de seleção de portfólios pode ser usado para complementar os resultados da teoria da fronteira eficiente, de modo a fornecer informações quantitativas mais precisas para auxiliar no processo decisório.
Tabela 4 - Resultados para retorno, riscos e EC dos portfólios.

\begin{tabular}{c|c|c|c|c|c}
\hline \multirow{2}{*}{ Portfólio } & \multirow{2}{*}{$\begin{array}{c}\text { Retorno } \\
\text { (US\$ }\end{array}$} & \multicolumn{2}{|c|}{$\begin{array}{c}\text { Considerando o } \\
\text { desvio-padrão }\end{array}$} & \multicolumn{2}{c}{$\begin{array}{c}\text { Considerando o semi- } \\
\text { desvio-padrão }\end{array}$} \\
\cline { 3 - 6 } & & EC (1) & EC (2) & EC (1) & EC (2) \\
\hline A1 & 100 & 92,01 & 93,15 & 88,83 & 90,69 \\
\hline A2 & 150 & 132,03 & 134,60 & 124,87 & 129,06 \\
\hline A3 & 200 & 168,05 & 172,62 & 155,32 & 162,77 \\
\hline A4 & 250 & 200,08 & 207,21 & 180,19 & 191,82 \\
\hline A5 & 300 & 228,12 & 238,39 & 199,47 & 216,23 \\
\hline A6 & 350 & 252,16 & 266,14 & 213,17 & 235,97 \\
\hline A7 & 400 & 272,21 & 290,47 & 221,28 & 251,07 \\
\hline A8 & 450 & 288,27 & 311,37 & 223,81 & 261,51 \\
\hline A9 & 500 & 300,33 & 328,85 & 220,75 & 267,29 \\
\hline A10 & 550 & 308,40 & 342,91 & 212,11 & 268,43 \\
\hline A11 & 600 & 312,47 & 353,55 & 197,88 & 264,90 \\
\hline A12 & 650 & 312,54 & 360,75 & 178,06 & 256,72 \\
\hline A13 & 700 & 308,16 & 364,14 & 152,04 & 243,37 \\
\hline A14 & 750 & 299,10 & 363,51 & 119,49 & 224,58 \\
\hline A15 & 800 & 285,36 & 358,88 & 80,40 & 200,33 \\
\hline A16 & 850 & 266,94 & 350,23 & 34,77 & 170,64 \\
\hline A17 & 900 & 243,84 & 337,57 & $-17,39$ & 135,51 \\
\hline A18 & 950 & 216,06 & 320,91 & $-76,10$ & 94,92 \\
\hline A19 & 1000 & 183,60 & 300,23 & $-141,34$ & 48,89 \\
\hline & & & & & \\
\hline
\end{tabular}

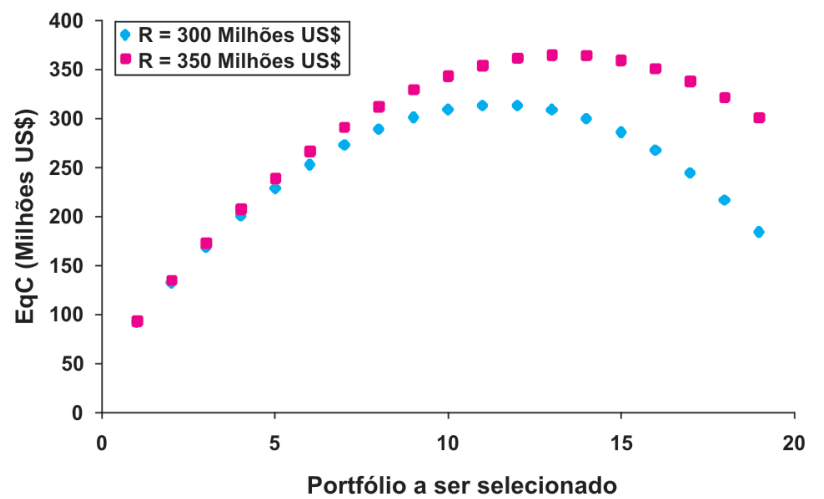

(b)

Figura 3 - Gráficos dos equivalentes certo para os portfólios utilizando (a) o desvio-padrão e o (b) semidesvio-padrão como métrica de risco. 
Rafael C. Galeno et al.

\section{Agradecimentos}

Os autores gostariam de agradecer o apoio financeiro da FAPESP, CNPq, PETROBRAS e do Centro de Estudos de Petróleo (CEPETRO) da Unicamp para a realização dessa pesquisa.

\section{Referências bibliográficas}

CAMPBELL, J. M., CAMPBELL, J. M., CAMPBELL, J. A. Analyzing and Managing Risky Investments. Oklahoma: John Campbell, 2001. 485p.

CAMPBELL, J. Y., LO, A.W., MacKINLAY,

A. C. The econometrics of financial markets. New Jersey: Princeton University Press, 1997. 611p.

COSTA LIMA, G. A. C., SCHIOZER, R. F., SUSLICK, S. B. The efficient frontier of oil projects portfólio as a tool for decision-making. In: Rio Oil \& Gas 2006: Expo and Conference, 2006. Rio de Janeiro. Anais do Rio Oil \& Gas Expo and Conference 2006.

COSTA LIMA, G. A. C., SUSLICK, S. B , PEREIRA, J.Q. Portfolio optimization of oil production projects using mathematical programming and utility theory. In: 18th International Congress of Mechanical Engineering, 2005. Ouro Preto. 11p.

COZZOLINO, J. M. Controlling risk in capital budgeting: a practical use of utility theory for measurement and control of petroleum exploration risk. The Engineering Economist, v.25, n. 3, p.161-186, 1980.

GALENO, R. C. Seleção de portfólios de projetos de exploração e produção de petróleo utilizando um modelo exponencial de aversão ao risco. Faculdade de Engenharia Mecânica, Universidade Estadual de Campinas, 2008. 115p. (Dissertação de Mestrado).

HOLLOWAY, C.A. Decision Making Under Uncertainty: Models and Choices. New Jersey: Prentice-Hall, 1979. 522p.

KRITZMAN, M. P. The portable financial analyst: what practitioners need to know. New Jersey: Wiley, 2003. 260p.

LUENBERGER, D. G. Investment science. Oxford: Oxford Press, 1998. 494p.

MARKOWITZ, H. M. Portfolio selection: efficient diversification of investments. New York: John Willey \& Sons, 1959. 343p

MARKOWITZ, H.M. Portfolio selection. Journal of Finance, v. 7, n. 1, p. 77-91, 1952.

NEPOMUCENO, F., SUSLICK, S. B. Alocação de recursos financeiros em projetos de risco na exploração de petróleo. Revista de Administração de Empresas (RAE - FGV), v. 40, n. 1, p. $63-76,2000$.

PETROBRAS. Relações com o investidor. Disponível em [http://www.petrobras.com.br]. Acessado em 20/09/2008.

SIDDIQUI, M., AL-YATEEM, K., AL-THAWADI, A. A new tool to evaluate the feasibility of petroleum exploration projects using a combination of deterministic and probabilistic methods. In: SPE Middle East Oil \& Gas Show and Conference, 2007. Bahrain. (Paper SPE, 105694).

SUSLICK, S.B., SCHIOZER, D.J. Risk analysis applied to petroleum exploration and production: an overview. Journal of Petroleum Science and Engineering, v. 44, n. 2, p.1-9, 2004.

VON NEUMANN, J., MORGENSTERN, O. The theory of games and economic behavior. Princeton: Princeton University Press, 1953. 648p.

WALLS, M. R. Combining decision analysis and portfolio management to improve project selection in the exploration and production firm. Journal of Petroleum Science and Engineering, v. 44, n. 7, p. 55-65, 2004.

WALLS, M. R. Corporate risk tolerance and capital allocation: a practical approach to implementing an exploration risk policy. Journal of Petroleum Technology, v. 47, n. 4, p. 307-311, 1995.

WALLS, M. R., DYER, J.S. Risk propensity and firm performance: a study of the petroleum exploration industry. Management Science, v. 42, n. 7, p. 1004-1021, 1996.

Artigo recebido em 12/10/2008 e aprovado em 18/05/2009.

\section{A REM tem novo endereço: FUNDAÇÃO GORCEIX - REM Rua Carlos Walter Marinho Campos, 57 Bairro: Vila Itacolomy 35400-000 - Ouro Preto - MG $\begin{array}{ll}\text { (31) } 3551-4730 & \text { (31) } 3559-7408\end{array}$} www.rem.com.br 